\title{
2017 Reviewer Acknowledgement
}

\author{
Editorial Office of Exploratory Research and Hypothesis in Medicine
}

We thank the following reviewers for their contribution and support in 2017.

\author{
Belgin Akan \\ Turkey \\ Majid Anushiravani \\ Iran \\ Alessandra Arcolaci \\ Italy \\ Marianne Besnard \\ France \\ Tatiana Betakova \\ Slovakia \\ Igor Burstyn \\ United States \\ Javier Camacho \\ Mexico \\ Bohao Chen \\ United States \\ Szu-ta Chen \\ China (Taiwan) \\ Jianding Cheng \\ China \\ Hsueh-Ling Cheng \\ China (Taiwan) \\ Salvatore Chirumbolo \\ Italy \\ Ahmet Cumaoglu \\ Turkey \\ Ina Danquah \\ Germany \\ Andrew Stewart Day \\ New Zealand \\ Bhavtosh Dedania \\ United States \\ Srdjan Denic \\ United Arab Emirates \\ Jianqiang Ding \\ China \\ Iliana B. Doycheva \\ Bulgaria \\ Mehmet Ali Ergun \\ Turkey \\ Ahmet Eroglu \\ Turkey \\ Yuchen Fan \\ China \\ Shirley Steffany Muñoz Fernández \\ Brazil
}

[DOI: 10.14218/ERHM.2017.000RA
Clara Gabás-Rivera

Spain

Xueqin Gao

United States

Shanzhong Gong

United States

Osamu Handa

Japan

Richard Hansen

United Kingdom

Lokesh Jain India

Wan Fariza Wan Jamaludin Malaysia

Cagatay Karaaslan Turkey

Aynur Karadag Turkey

A. Kauppinen

Finland

Abbas Khani

Switzerland

Chao Li

United States

Dong Li

China

Junxia Li

China

José Manuel Lou-Bonafonte Spain

Mark Lucock Australia

Charlotte Martin Australia

Tracey A. Martin United Kingdom

Gerald Martone United States

David Miller

United Kingdom

John H. Miller

New Zealand

Rosiane A. Miranda Brazil

Javier A. Miret Spain

Slávka Mrosková

Slovakia

Ashesh Nandy

India
Nenad Naumovski

Australia

Yoshikazu Ogawa Japan

Barbara Olendzki United States

Keith Pecor United States

Petrick Periyasamy Malaysia

Aline Valeska Probst France

Edward V. Quadros United States

Aleksei V Rakov United States

Rama Sankar Rath India

Samiran Ray United Kingdom

Jonathan Roth Israel

Ian Russell United Kingdom

Miroslav Simunic Croatia

Keerti Singh Barbados

Surajit Sinha United States

H Skaltsa Greece

James Stamey United States

Deborah Sundin Australia

Bartlomiej Szulczyk Poland

Pawel Szulczyk Poland

Jonathan E. Teitelbaum United States

Georgios Tsoulfas Greece

Tuxun Tuerhongjiang China

Alexander M. Vaiserman Ukraine

Guojun Wang United States 
Lili Wang

United States

Toru Watanabe

Japan

Ewa Widy-Tyszkiewicz

Poland

Jennifer M. Windt

Australia

Qichao Wu

China

Guogang Xu

China
Yongkang Yang

United States

Şenay Görücü Yılmaz

Turkey

Juliana Yordanova

Germany

Chengyue Zhang

United States

Sarah Zhang

China

Huantian Zhang

China

\section{Chong Zhang}

China

Weilin Zhang

China

Lanjing Zhang

United States

Lei Zhong

Uruguay

Xiaohong Zhou

United States 